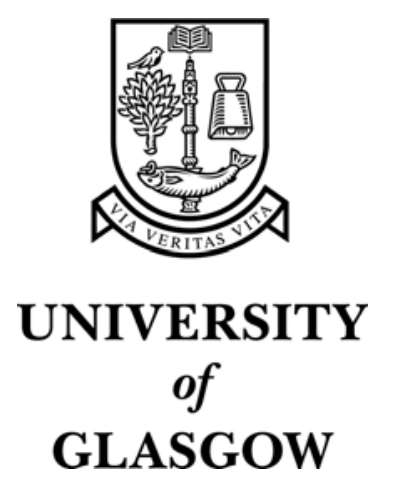

Keston, D.A. and Laing, E.W. and Diver, D.A. (2003) Bernstein modes in a weakly relativistic electron-positron plasma. Physical Review E 67:art 036403.

http://eprints.gla.ac.uk/1618/ 


\title{
Bernstein modes in a weakly relativistic electron-positron plasma
}

\author{
D A Keston, E W Laing, and D A Diver \\ Dept of Physics and Astronomy, Kelvin Building, \\ University of Glasgow, Glasgow G12 8QQ, Scotland UK.*
}

(Dated: March 30, 2004)

\begin{abstract}
The form of the propagating electrostatic Bernstein mode in a relativistic electron-positron plasma is markedly different from that in the classical plasma, once the momentum-dependent cyclotron frequency is accounted for in full inside the integrations. Given that particles in different parts of momentum space 'see' a different cyclotron frequency, there is no simple global singularity which reproduces the classical dispersion features.
\end{abstract}

PACS numbers: 52.25.Dg, 52.25.Xz, 52.27.Ep, 52.27.Ny, 52.35.Fp

\section{INTRODUCTION}

Astrophysical electron-positron plasmas are, by their nature, highly energetic. The mathematical modelling of such media must be relativistic, since the thermal content of such a plasma will be significant compared to the particles' rest energy $[1,2]$. In this paper we describe the kinetic theory of weakly relativistic plasmas, defined to be those for which the equilibrium distribution function is taken to be Maxwellian, but the full relativistic correction for the mass-dependent cyclotron frequency is included.

We present new dispersion curves for the particular case of Bernstein modes [3-5], which are electrostatic waves propagating perpendicularly, and nearly perpendicularly, to the uniform equilibrium magnetic field. In this article we will concentrate on the specific case of perpendicular propagation. The classical treatment of these modes for an electron-ion plasma depends upon an arbitrarily low temperature, and yet is not entirely consistent with cold plasma theory [6-8]. Part of the discrepancy undoubtedly can be attributed to the handling of singularities in the classical kinetic case, in which the harmonic resonances are removed from the integration over particle distribution. In fact, the correct method of treating these points is to recognise that the cyclotron frequency is momentum dependent (that is, the cyclotron frequency drops as the particle momentum increases), and treat the singular integral accordingly. Other researchers have produced purely formal solutions [2] for an arbitrary relativistic $\gamma$ but without any phenomenological analysis, or have tackled either ultra-relativistic plasmas $[9,10]$ or weakly relativistic behaviour in which $\gamma \approx 1+p^{2} /\left(2 m^{2} c^{2}\right)[6-9]$; most of these treatments are for electron-ion plasmas, and assume a stationary ion background. In this article we present a treatment of the Bernstein modes for a weakly relativistic electronpositron plasma, valid for moderate values of $\gamma$, and without the 'stationary ion' approximation. The next section gives the full formal statement of the dispersion relation

*enquiries to d.diver@physics.gla.ac.uk for all linear Vlasov-kinetic modes in an $e^{+} e^{-}$plasma. Section III describes the non-relativistic Bernstein modes in this context, to provide an essential comparison for the weakly relativistic case in the subsequent section.

\section{MODEL EQUATIONS}

The starting point is the Vlasov equation formulated in momentum space, rather than velocity space [9]:

$$
\frac{\partial f_{s}}{\partial t}+\boldsymbol{v} \cdot \frac{\partial f_{s}}{\partial \boldsymbol{r}}+q_{s}(\boldsymbol{E}+\boldsymbol{v} \times \boldsymbol{B}) \cdot \frac{\partial f_{s}}{\partial \boldsymbol{p}}=0
$$

where $f_{s}(\boldsymbol{r}, \boldsymbol{p}, t)$ and $q_{s}$ are respectively the particle distribution function and charge, for particles of species $s$, where $s$ is either $e$ for electrons, or $p$ for positrons. Together with Maxwell's equations

$$
\begin{aligned}
\nabla \times \boldsymbol{B} & =\mu_{0} \sum_{s} n_{s} \int q_{s} \boldsymbol{v} f_{s} \mathrm{~d} \boldsymbol{p}+\frac{1}{c^{2}} \frac{\partial \boldsymbol{E}}{\partial t} \\
\nabla \times \boldsymbol{E} & =\frac{\partial \boldsymbol{B}}{\partial t} \\
\nabla \cdot \boldsymbol{B} & =0 \\
\nabla \cdot \boldsymbol{E} & =\sum_{s} \frac{q_{s}}{\epsilon_{0}} n_{s} \int f_{s} \mathrm{~d} \boldsymbol{p}
\end{aligned}
$$

Eqs. (1)-(5) constitute a complete set for describing the plasma behaviour (although Eq. (4) and Eq. (5) can be considered as initial conditions only). Note that $n_{s}$ is the number density of species $s$.

Consider now the linearised equations appropriate for describing small amplitude waves. Assuming that the equilibrium quantities are distinguished from perturbations by a subscript 0 , we have

$$
\begin{gathered}
\left(\boldsymbol{p} \times \boldsymbol{B}_{0}\right) \cdot \frac{\partial f_{0 s}}{\partial \boldsymbol{p}}=0 \\
\frac{\partial f_{s}}{\partial t}+\boldsymbol{v} \cdot \frac{\partial f_{s}}{\partial \boldsymbol{r}}+q\left(\boldsymbol{v} \times \boldsymbol{B}_{0}\right) \cdot \frac{\partial f_{s}}{\partial \boldsymbol{p}} \\
=-q(\boldsymbol{E}+\boldsymbol{v} \times \boldsymbol{B}) \cdot \frac{\partial f_{0 s}}{\partial \boldsymbol{p}}
\end{gathered}
$$




$$
\nabla \times \boldsymbol{B}=\mu_{0} \sum_{s} \frac{q_{s}}{m_{s}} n_{s} \int \boldsymbol{p} f_{s} \mathrm{~d} \boldsymbol{p}+\frac{1}{c^{2}} \frac{\partial \boldsymbol{E}}{\partial t}
$$

Our interest lies in small-amplitude waves, and so we assume that the equilibrium is uniform in space, and that perturbations vary as $\exp i(\boldsymbol{k} \cdot \boldsymbol{r}-\omega t)$. The full details of the solution procedure are well documented elsewhere $[5,11,12]$ and need not be repeated. After some manipulation the dispersion relation for small amplitude waves can be written in the form

$$
\begin{aligned}
& \boldsymbol{k} \times(\boldsymbol{k} \times \boldsymbol{E})+\frac{\omega^{2}}{c^{2}} \boldsymbol{E}= \\
& -\frac{2 \pi}{c^{2}} \sum_{s} \omega_{p s}^{2} \int_{0}^{\infty} \int_{-\infty}^{\infty} \mathrm{d} p_{\perp} \mathrm{d} p_{\|} \sum_{n=-\infty}^{\infty} \mathcal{Q}_{n s} \cdot \boldsymbol{E}
\end{aligned}
$$

in which cylindrical co-ordinates $\left(p_{\|}, p_{\perp}, \phi\right)$ for the momentum have been used, with $\|, \perp$ denoting the direction parallel, and perpendicular, to the equilibrium magnetic field, and where $\omega_{p s}=n_{s} q_{s}^{2} /\left(\epsilon_{0} m_{s}\right)$ denotes the plasma frequency of species $s$ with (rest) mass $m_{s}$. Note that the summation is over integer $n$, and that we have assumed that the equilibrium distribution function $f_{s 0}$ is isotropic, so that $p_{\|} \partial f_{s 0} / \partial p_{\perp}=p_{\perp} \partial f_{s 0} / \partial p_{\|}$. The matrix $\mathcal{Q}_{n s}$ is defined by

$$
\begin{aligned}
\mathcal{Q}_{n s}= & U_{s}\left(\omega-k_{\|} v_{\|}-n \Omega_{s}\right)^{-1} \times \\
& {\left[\begin{array}{ccc}
p_{\perp}^{2} \frac{n^{2}}{\zeta_{s}^{2}} J_{n}^{2} & -\mathrm{i} p_{\perp}^{2} \frac{n}{\zeta_{s}} J_{n} J_{n}^{\prime} & p_{\perp} p_{\|} \frac{n}{\zeta_{s}} J_{n}^{2} \\
\mathrm{i} p_{\perp}^{2} \frac{n}{\zeta_{s}} J_{n} J_{n}^{\prime} & p_{\perp}^{2} J_{n}^{\prime 2} & \mathrm{i} p_{\perp} p_{\|} J_{n}^{\prime} J_{n} \\
p_{\perp} p_{\|} \frac{n}{\zeta_{s}} J_{n}^{2} & -\mathrm{i} p_{\|} p_{\perp} J_{n}^{\prime} J_{n} & p_{\|}^{2} J_{n}^{2}
\end{array}\right] }
\end{aligned}
$$

in which $\Omega_{s}=q_{s} B_{0} / m_{s}$ is the cyclotron frequency of species $s, m_{s}$ is the particle mass, $J_{n}$ is the Bessel function of order $n$ and argument $\zeta_{s}$, and the following definitions apply:

$$
\begin{aligned}
U_{s} & =\left(\omega-k_{\|} v_{\|}\right) \frac{\partial f_{0 s}}{\partial p_{\perp}}+k_{\|} v_{\perp} \frac{\partial f_{0 s}}{\partial p_{\|}} \\
\zeta_{s} & =\frac{k_{\perp} v_{\perp}}{\Omega_{s}}
\end{aligned}
$$

There are several simplifications that can be applied immediately. Since we wish to study perpendicular Bernstein modes primarily, $k_{\|}=0$. Next, noting that $\zeta_{e}=$ $-\zeta_{p}$, the sum over species can be carried out quite simply given that all the elements of $\mathcal{Q}_{n s}$ are identical in magnitude for an electron-positron plasma, with only the $(1,3)$, $(2,3),(3,1)$ and $(3,2)$ elements changing sign. Hence when the positron and electron matrices are added, the result is

$$
\mathcal{Q}_{n p}+\mathcal{Q}_{n e}=2 \frac{\omega^{2}}{\Delta_{n}} \frac{\partial f_{0}}{\partial p_{\perp}} \times\left[\begin{array}{ccc}
p_{\perp}^{2} \frac{n^{2}}{\zeta^{2}} J_{n}^{2} & -\mathrm{i} p_{\perp}^{2} \frac{n}{\zeta} J_{n} J_{n}^{\prime} & p_{\perp} p_{\|} \frac{n^{2}}{\zeta} \frac{\Omega}{\omega} J_{n}^{2} \\
\mathrm{i} p_{\perp}^{2} \frac{n}{\zeta} J_{n} J_{n}^{\prime} & p_{\perp}^{2} J_{n}^{\prime 2} & \mathrm{i} p_{\perp} p_{\|} \frac{n \Omega}{\omega} J_{n}^{\prime} J_{n} \\
p_{\perp} p_{\|} \frac{n^{2}}{\zeta} \frac{\Omega}{\omega} J_{n}^{2} & -\mathrm{i} p_{\perp} p_{\|} \frac{n \Omega}{\omega} J_{n}^{\prime} J_{n} & p_{\|}^{2} J_{n}^{2}
\end{array}\right]
$$

in which $\Delta_{n}=\omega^{2}-n^{2} \Omega^{2}=\omega^{2}-n^{2} \Omega_{0}^{2} / \gamma^{2}$ and all speciesrelated subscripts have been dropped, the positron value of any quantity being assumed. A further simplification results from considering the summing over integer $n$, since those matrix elements which contain only a linear factor $n$ must vanish, given that $n$ ranges over all positive and negative integer values. Finally, note that since the integration in $p_{\|}$ranges from $-\infty$ to $\infty$, those elements which are odd in $p_{\|}$vanish identically on inte- gration, and therefore may be discarded. As a result, the right-hand side of Eq. (9) can be written

$$
-4 \pi \omega_{p}^{2} \frac{\omega^{2}}{c^{2}} \sum_{n=-\infty}^{\infty} \int_{-\infty}^{\infty} \mathrm{d} p_{\|} \int_{0}^{\infty} \mathrm{d} p_{\perp} \mathcal{K}_{n} \cdot \boldsymbol{E}
$$


where

$$
\mathcal{K}_{n}=\frac{1}{\Delta_{n}} \frac{\partial f_{0}}{\partial p_{\perp}}\left[\begin{array}{ccc}
p_{\perp}^{2} \frac{n^{2}}{\zeta^{2}} J_{n}^{2} & 0 & 0 \\
0 & p_{\perp}^{2} J_{n}^{\prime 2} & 0 \\
0 & 0 & p_{\|}^{2} J_{n}^{2}
\end{array}\right]
$$

Note that the left-hand-side of Eq. (9) simplifies to

$$
\left[\begin{array}{ccc}
\frac{\omega^{2}}{c^{2}} & 0 & 0 \\
0 & \frac{\omega^{2}}{c^{2}}-k_{\perp}^{2} & 0 \\
0 & 0 & \frac{\omega^{2}}{c^{2}}-k_{\perp}^{2}
\end{array}\right] \cdot \boldsymbol{E}
$$

showing that for the particular case of wave propagation perpendicular to the equilibrium magnetic field in an equal-mass plasma, the kinetic modes simplify beautifully. Since this article is concerned with Bernstein modes, only the solution for non-zero $E_{x}$ will be examined, for which the dispersion relation is

$$
1=-4 \pi \omega_{p}^{2} \sum_{n=-\infty}^{\infty} \int_{-\infty}^{\infty} \mathrm{d} p_{\|} \int_{0}^{\infty} \mathrm{d} p_{\perp} \frac{\partial f_{0}}{\partial p_{\perp}} \frac{p_{\perp}^{2} n^{2}}{\Delta_{n} \zeta^{2}} J_{n}^{2}(\zeta)
$$

However, in order to progress, the equilibrium distribution function $f_{0}$ must be specified, and this is the subject of the next sections.

\section{CLASSICAL, NON-RELATIVISTIC BERNSTEIN MODES}

In the usual, classical treatment of electron Bernstein modes for a plasma with stationary ions, the relativistic terms are discarded, and the equilibrium distribution function is the classical Maxwell-Boltzmann one,

$$
f_{0}(\boldsymbol{p})=\left(2 \pi m_{e} k_{B} T\right)^{-3 / 2} \exp \left[-p^{2} /\left(2 m_{e} k_{B} T\right)\right]
$$

which is written here in momentum form, and in which $m_{e}$ is the electron (and positron) rest mass. It is instructive to proceed with the fully non-relativistic calculation for an electron-positron plasma, since this calculation will serve as a vital contrast to the relativistic treatment that follows. The most important aspect of the non-relativistic calculation is that the particle mass remains a constant, and therefore the cyclotron frequency is not momentum-dependent. Considering the $(1,1)$ th entry of the matrices in Eq. (16) and Eq. (15), the dispersion relation for Bernstein waves can be written

$$
\begin{aligned}
& 1=4 \pi(2 \pi)^{-3 / 2}\left(m_{e} k_{B} T\right)^{-5 / 2} \omega_{p}^{2} \times \\
& \sum_{n=-\infty}^{\infty} \frac{n^{2}}{\Delta_{n}} \int_{-\infty}^{\infty} \mathrm{d} p_{\|} \int_{0}^{\infty} \mathrm{d} p_{\perp} \frac{p_{\perp}^{3}}{\zeta^{2}} J_{n}^{2}(\zeta)
\end{aligned}
$$

Notice that $\Delta_{n}$ can be taken outside the integration, since there is no relativistic correction to the cyclotron

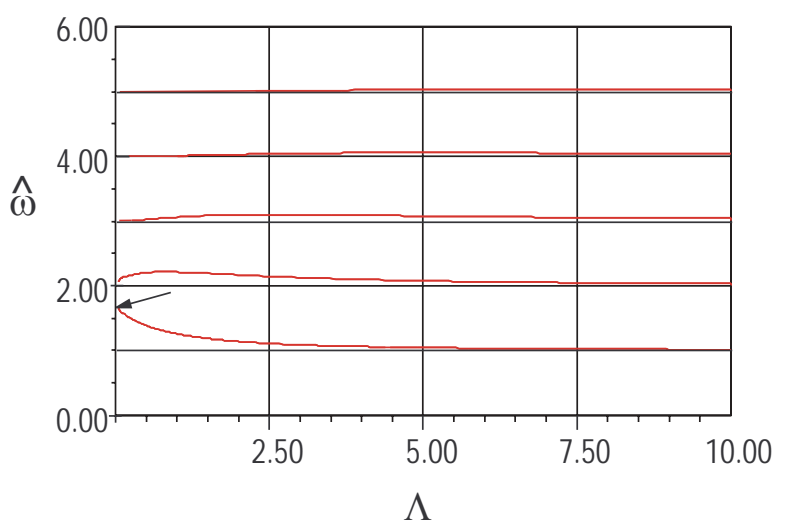

FIG. 1: Dispersion curves showing solutions in $\hat{\omega}-\Lambda$ space for the classical, non-relativistic case, where $\hat{\omega}=\omega / \Omega$. The vertical axis is $\hat{\omega}$, and the horizontal, $\Lambda$. The non-dimensional hybrid frequency is $\sqrt{3}$, for $\hat{\omega}_{p}^{2}=\omega_{p}^{2} / \Omega^{2}=1$, and is shown arrowed in the figure.

frequency. In fact, the double integration can be performed analytically, since the integrations with respect to $p_{\|}$and $p_{\perp}$ are independent. The result is the form of the dispersion relation

$$
\Lambda=4 \omega_{p}^{2} \exp (-\Lambda) \sum_{n=1}^{\infty} \frac{n^{2}}{\omega^{2}-n^{2} \Omega^{2}} I_{n}(\Lambda)
$$

where $\Lambda$, the ratio of thermal energy to wave energy, is given by

$$
\Lambda=\frac{k_{\perp}^{2} k_{B} T}{m_{e} \Omega^{2}}
$$

Eq. (20) is exact for an electron-positron plasma; there is no 'static ion' approximation here. Solutions occur at frequencies close to the cyclotron harmonics, and the full curves are presented in Figs. 1 and 2, which show the cases $\omega_{p} / \Omega=1$ and $\omega_{p} / \Omega=3$ respectively. Note that Eq. (20) also gives the approximate dispersion relation for an electron plasma with stationary ions, if the factor 4 is replaced by 2 .

\section{A. Behaviour for small $\Lambda$}

A deeper insight into the characteristics of the dispersion relation can be gleaned from the the Taylor expansion of Eq. (20) for small $\Lambda$, which gives the longwavelength (or low temperature) approximate dispersion relation

$$
\Lambda \approx \frac{\Delta_{H} \Delta_{2}}{6 \Omega^{2} \omega_{p}^{2}}, \quad \Lambda \ll 1
$$

in which

$$
\Delta_{H}=\omega^{2}-\omega_{H}^{2}=\omega^{2}-2 \omega_{p}^{2}-\Omega^{2}
$$




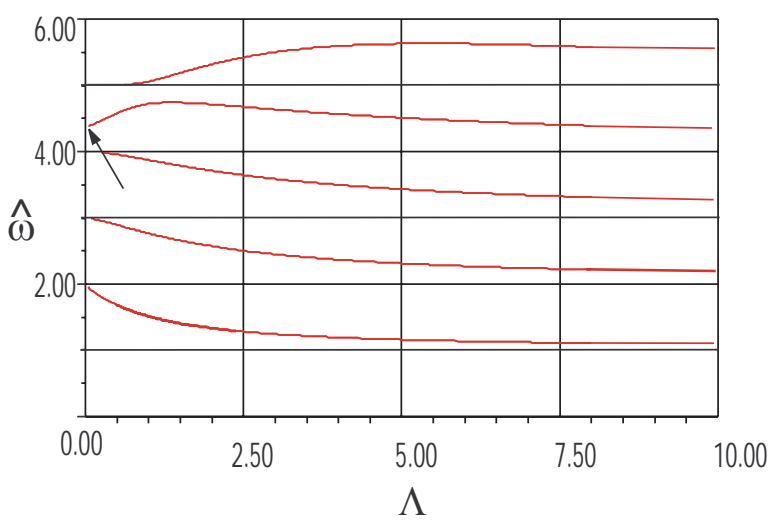

FIG. 2: Dispersion curves showing solutions in $\hat{\omega}-\Lambda$ space for the classical, non-relativistic case, where $\hat{\omega}=\omega / \Omega$. The vertical axis is $\hat{\omega}$, the horizontal, $\Lambda$. The non-dimensional hybrid frequency is $\sqrt{19}$, for $\hat{\omega}_{p}=\omega_{p} / \Omega=3$, and is shown arrowed in the figure.

defining the hybrid frequency $\omega_{H}$. First, note there is no solution for $\omega=\Omega, \quad \Lambda \ll 1$, since for this case Eq. (22) predicts $\Lambda \approx 1$, a contradiction. If $\omega^{2}=\omega_{H}^{2}+\epsilon$, then

$$
\Lambda \approx \frac{2 \omega_{p}^{2}-3 \Omega^{2}}{6 \omega_{p}^{2} \Omega^{2}} \epsilon
$$

If $2 \omega_{p}^{2}<3 \Omega^{2}$, so that $\omega_{H}<2 \Omega$, then Eq. (24) shows that $\epsilon<0$ is required to ensure $\Lambda>0$; hence the dispersion curve shows $\omega$ dropping as $\Lambda$ increases from 0 . The next possible solution is one for which $\omega^{2}=4 \Omega^{2}+\epsilon$, giving the local dispersion relation

$$
\Lambda \approx \frac{3 \Omega^{2}-2 \omega_{p}^{2}}{6 \omega_{p}^{2} \Omega^{2}} \epsilon
$$

In this case, assuming again $\omega_{H}<2 \Omega$, the dispersion curve shows that $\omega$ increases as $\Lambda$ increases. Hence, for $\omega_{H}<2 \Omega$, there is no wave solution for frequencies between $\omega_{H}$ and $2 \Omega$.

If, on the other hand, $2 \omega_{p}^{2}>3 \Omega^{2}$, so that $\omega_{H}>2 \Omega$, the dispersion curve at $\omega=2 \Omega$ shows $\omega$ decreasing as $\Lambda$ increases from zero. In general, there is no long-wavelength mode in the approximate frequency interval starting just above $\omega_{H}$ and ending before the next highest cylcotron harmonic.

\section{B. Behaviour for large $\Lambda$}

For very short wavelength solutions, given that

$$
\exp (-\Lambda) I_{n}(\Lambda) \sim(2 \pi \Lambda)^{-1 / 2}, \quad \Lambda \gg 1
$$

then an approximate dispersion relation

$$
\Lambda^{3 / 2} \approx \frac{4 \omega_{p}^{2}}{\sqrt{2 \pi}} \sum_{n=1}^{\infty} \frac{n^{2}}{\omega^{2}-n^{2} \Omega^{2}}, \quad \Lambda \gg 1
$$

shows that short wavelength solutions can only occur at frequencies close to cyclotron harmonics: $\omega \approx n \Omega$.

\section{Stationary modes}

Solutions to the dispersion relation for frequencies higher than the hybrid frequency have the property that the group velocity has a zero for finite values of $\Lambda$, since the dispersion curves are bell-shaped[5]. From the curves in Figs. 1 and 2, the maxima in the dispersion curve occur at intermediate values of $\Lambda$, and so neither of the two approximate treatments described above is adequate to reveal this effect. Instead, if

$$
f_{n}(\Lambda)=\frac{e^{-\Lambda}}{\Lambda} I_{n}(\Lambda)
$$

then the full dispersion relation can be written in the form

$$
1=4 \omega_{p}^{2}\left[\frac{f_{1}(\Lambda)}{\omega^{2}-\Omega^{2}}+\frac{4 f_{2}(\Lambda)}{\omega^{2}-4 \Omega^{2}}+\ldots\right]
$$

For illustrative purposes, we shall consider only the first 2 terms, with the assumption $\Omega<\omega_{H}<2 \Omega$, as before. An excellent approximation to $f_{n}(x)$ is

$$
f_{n}(x) \approx 2^{-n} x^{n-1}\left(\frac{1}{n !}+\frac{x^{2}}{4(n+1) !}\right) e^{-x}
$$

For solutions near the second harmonic, that is $\omega^{2}=$ $4 \Omega^{2}+\epsilon, \epsilon>0$ the position where the tangent to the disperion curve is zero is readily approximated by the solution to

$$
f_{2}^{\prime}(\Lambda) \approx 0
$$

which, on using Eq. (30), yields the cubic equation

$$
\Lambda^{3}-3 \Lambda^{2}+12 \Lambda-12=0
$$

the roots of which are the values of $\Lambda$ for which the tangent vanishes. In fact Eq. (32) has only one real positive root, at $\Lambda_{0} \approx 1.22$, in close agreement with Figs. 1 and 2. A more detailed asymptotic analysis[5] places these critical points at $\Lambda=1.25,3.05,5.44$ and 8.46 for frequencies near $\hat{\omega}=2,3,4$, and 5 , respectively. Given that $\Lambda \approx k_{\perp}^{2} R_{L}^{2} / 3$, where $R_{L}$ is the Larmor radius these critical values correspond to $k_{\perp} R_{L} \approx n, n=2,3,4$ revealing a resonance between the wavelength of the Bernstein mode and the influence of the magnetic field via the Larmor radius.

Hence all solutions for frequencies higher than the hybrid frequency have bell-shaped dispersion curves, showing that for a given frequency, there are either two solutions, corresponding to different values of $k_{\perp}$, or no solution at all, if the frequency is sufficiently far from a cyclotron harmonic to be in the band-gap. Note also that 
there is always one $\left(\omega, k_{\perp}\right)$ pair for which the group velocity is zero, and is therefore a non-propagating wave. However, for all frequencies less than the hybrid frequency, there is always a unique value of $k_{\perp}$ giving an electrostatic wave (allowing for infinite $\Lambda$ values); none of these solutions has zero group velocity.

\section{Summary}

The analysis in this section shows how the classical, non-relativistic Bernstein modes can be described wholly analytically, with general dispersion relations expressible in closed form. This is only possible because the integrations can be performed independently, since the poles in the denominator are not functions of momentum.

\section{WEAKLY RELATIVISTIC APPROXIMATION}

In the relativistic case, the cyclotron frequency is a function of momentum, and the integrations over $p_{\|}$and $p_{\perp}$ are not independent, as can be seen from the true form of the relativistic equilibrium function $[2,9,13]$ :

$$
f_{0}(\boldsymbol{p})=\left(4 \pi m_{e}^{3} c^{3}\right)^{-1} \frac{a}{K_{2}(a)} \exp (-a \gamma)
$$

where

$$
a=\frac{m_{e} c^{2}}{k_{B} T}
$$

is the non-dimensional reciprocal relativistic temperature, $K_{2}$ is the modified Bessel function of the second kind, of order 2 , and

$$
\gamma=\left[1+\frac{p_{\perp}^{2}+p_{\|}^{2}}{m_{e}^{2} c^{2}}\right]^{1 / 2}
$$

is the usual relativistic Lorentz factor. It is clear that on substituting Eq. (33) into Eq. (17) there is no possibility of separating the integrand into independent parts, nor of extracting $\Delta_{n}$ from any part of the integration. As a physically meaningful compromise, we define the weakly relativistic approximation to be one in which the equilibrium distribution function for positrons and electrons is the classical Maxwellian given by Eq. (18), but in which the Lorentz factor $\gamma$ is retained in full in the $\Delta_{n}$ term in the denominator of Eq. (14). This simplifies the integration over momentum-space, without sacrificing the significant relativistic Doppler correction to the singularity. Note that $\gamma$ will not be expanded binomially as in other articles $[6,8]$.

To simplify the algebra, the following non-dimensional variables are adopted from now on:

$$
\begin{array}{cc}
\hat{\omega}=\omega / \Omega_{0} & \hat{\omega}_{p}=\omega_{p} / \Omega_{0} \\
\hat{p}_{\|}=p_{\|} /\left(m_{e} c\right) & \hat{p}_{\perp}=p_{\perp} /\left(m_{e} c\right) \\
\hat{k}_{\perp}=k_{\perp} c / \Omega_{0} & \zeta=\hat{k}_{\perp} \hat{p}_{\perp}
\end{array}
$$

where $\Omega_{0}=e B_{0} / m_{e}$ is the rest cyclotron frequency, a constant. The full dispersion relation for weakly relativistic Bernstein modes can then be written in the form

$$
\begin{gathered}
\hat{\omega}^{2}=\frac{4 \hat{\omega}_{p}^{2}}{\sqrt{2 \pi} \hat{k}_{\perp}^{2}} a^{5 / 2} \sum_{n=1}^{\infty} \int_{0}^{\infty} \mathrm{d} \hat{p}_{\perp} \int_{-\infty}^{\infty} \mathrm{d} \hat{p}_{\|} \frac{n^{2} \gamma^{2} \hat{p}_{\perp}}{\gamma^{2}-n^{2} / \hat{\omega}^{2}} \times \\
J_{n}^{2}\left(\hat{k}_{\perp} \hat{p}_{\perp}\right) \exp \left[-\frac{1}{2} a\left(\hat{p}_{\perp}^{2}+\hat{p}_{\|}^{2}\right)\right]
\end{gathered}
$$

Noting that

$$
\frac{\gamma^{2}}{\gamma^{2}-n^{2} / \hat{\omega}^{2}}=1+\frac{n^{2}}{\hat{\omega}^{2}}\left(\frac{1}{\gamma^{2}-n^{2} / \hat{\omega}^{2}}\right)
$$

the integrand in Eq. (37) can be split into two parts, one of which has singularity. The first double integral can be performed wholly analytically to yield

$$
\begin{aligned}
& \int_{0}^{\infty} \int_{-\infty}^{\infty} \mathrm{d} \hat{p}_{\perp} \mathrm{d} \hat{p}_{\|} \hat{p}_{\perp} J_{n}^{2}\left(\hat{k}_{\perp} \hat{p}_{\perp}\right) \exp \left(-\frac{1}{2} a \hat{p}^{2}\right)= \\
& \sqrt{(}(2 \pi) a^{-3 / 2} e^{-\Lambda} I_{n}(\Lambda)
\end{aligned}
$$

where $\hat{p}^{2}=\hat{p}_{\perp}^{2}+\hat{p}_{\|}^{2}$. Moreover, the summation over index $n$ applied to Eq. (39) can be performed analytically, via

$$
\sum_{n=1}^{\infty} n^{2} I_{n}(\Lambda)=\frac{1}{2} \Lambda e^{\Lambda}
$$

allowing the full dispersion relation to be written in the form

$$
\hat{\omega}^{2}=2 \hat{\omega}_{p}^{2}+\frac{4 \hat{\omega}_{p}^{2} a^{5 / 2}}{\sqrt{2 \pi} \hat{\omega}^{2} \hat{k}_{\perp}^{2}} \sum_{n=1}^{\infty} n^{4} \int_{0}^{\infty} \mathrm{d} \hat{p}_{\perp} \hat{p}_{\perp} J_{n}^{2}\left(\hat{k}_{\perp} \hat{p}_{\perp}\right) e^{-a \hat{p}_{\perp}^{2} / 2} \times \int_{-\infty}^{\infty} \mathrm{d} \hat{p}_{\|} \frac{e^{-a \hat{p}_{\|}^{2} / 2}}{1+\hat{p}_{\|}^{2}+\hat{p}_{\perp}^{2}-n^{2} / \hat{\omega}^{2}}
$$


the $\hat{p}_{\|}$integral if $n^{2} / \hat{\omega}^{2}>1$, since $\gamma \geq 1$. Such singular integrals, where they occur, are properly defined by the procedure discussed below, and in the Appendix.

In particular, defining

$$
b_{n}^{2}=n^{2} / \hat{\omega}^{2}-1
$$

there are two clear cases that can be identified for $\hat{\omega}<n$, that is, $b_{n}^{2}>0$ :

$$
\begin{aligned}
& \text { 1. } b_{n}^{2}>\hat{p}_{\perp}^{2} \\
& \text { 2. } b_{n}^{2}<\hat{p}_{\perp}^{2}
\end{aligned}
$$

The singularities on the real line in the $\hat{p}_{\|}$integration arise for small enough $\hat{p}_{\perp}$, and so can be negotiated by splitting the range of the $\hat{p}_{\perp}$ integration into 2 parts, corresponding to cases (1) and (2): (1) 0 to $b_{n}$, and (2) $b_{n}$ to $\infty$. Only the first of these integration ranges incurs singularities on the real $\hat{k}_{\|}$line. In case (2), the denominator does not have zeros on the real $\hat{k}_{\|}$line, and so no singularity arises in the $\hat{p}_{\|}$integration. Finally, note that if $b_{n}^{2}<0$, then there are no zeros in the denominator, and the $\hat{p}_{\|}$integration is again well defined; this will be referred to as case (3).

Returning to the full dispersion relation Eq. (41) we can state that for cases (1) and (2), the double integration term can be written in the form

$$
\begin{aligned}
& \int_{0}^{b_{n}} \mathrm{~d} \beta \beta J_{n}^{2}\left(\hat{k}_{\perp} \sqrt{b_{n}^{2}-\beta^{2}}\right) e^{-\frac{1}{2}\left(b_{n}^{2}-\beta^{2}\right)} \mathcal{I}_{1}(a, \beta) \\
+ & \int_{0}^{\infty} \mathrm{d} \delta \delta J_{n}^{2}\left(\hat{k}_{\perp} \sqrt{\delta^{2}+b_{n}^{2}}\right) e^{-\frac{1}{2}\left(b_{n}^{2}+\delta^{2}\right)} \mathcal{I}_{2}(a, \delta)
\end{aligned}
$$

where the $\hat{p}_{\perp}$ integration has been transformed using the changes of variable $\hat{p}_{\perp}^{2}=b_{n}^{2}-\beta^{2}$ in the first integral, and $\hat{p}_{\perp}^{2}=b_{n}^{2}+\delta^{2}$ in the second. The $\hat{p}_{\|}$integration is folded inside the integrals $\mathcal{I}_{1,2}$, with the following definitions. Taking case (1) first,

$$
\begin{aligned}
\mathcal{I}_{1}(a, b) & =\int_{-\infty}^{\infty} \mathrm{d} z \frac{\exp \left(-a z^{2} / 2\right)}{z^{2}-b^{2}} \\
& =i \frac{\pi}{b} e^{-a b^{2} / 2} \operatorname{erf}\left(i \sqrt{a b^{2} / 2}\right)
\end{aligned}
$$

The integrand contains simple poles at $\pm b$, and the details of the requisite integration contour and subsequent evaluation of the integral are deferred to the Appendix, rather than quoted here. The second integral $\mathcal{I}_{2}$ is defined by

$$
\begin{aligned}
\mathcal{I}_{2}(a, b) & =\int_{-\infty}^{\infty} \mathrm{d} z \frac{\exp \left(-a z^{2} / 2\right)}{z^{2}+b^{2}} \\
& =\frac{\pi}{b} e^{a b^{2} / 2} \operatorname{erfc}\left(\sqrt{a b^{2} / 2}\right)
\end{aligned}
$$

where once again the full details of the evaluation of this integral are deferred to the Appendix.

With Eq. (44) and Eq. (45) substituted into Eq. (43), and with a final change of variable that simplifies the error function argument, the full dispersion relation Eq. (41) can be written in the form

$$
\hat{\omega}^{2}-2 \hat{\omega}_{p}^{2}=D \sum_{n=1}^{\infty} n^{4} \times \begin{cases}A_{n}^{\mathrm{I}}+A_{n}^{\mathrm{II}} & n \geq \hat{\omega} \\ A_{n}^{\mathrm{III}} & n<\hat{\omega}\end{cases}
$$

where

$$
\begin{aligned}
& A_{n}^{\mathrm{I}}=e^{-x_{n}^{2}} \int_{0}^{x_{n}} J_{n}^{2}\left(\kappa \sqrt{x_{n}^{2}-x^{2}}\right) i \operatorname{erf}(i x) \mathrm{d} x \\
& A_{n}^{\mathrm{II}}=e^{-x_{n}^{2}} \int_{0}^{\infty} J_{n}^{2}\left(\kappa \sqrt{x_{n}^{2}+x^{2}}\right) \operatorname{erfc}(x) \mathrm{d} x
\end{aligned}
$$

and

$$
A_{n}^{\mathrm{III}}=e^{-x_{n}^{2}} \int_{\left|x_{n}\right|}^{\infty} J_{n}^{2}\left(\kappa \sqrt{x_{n}^{2}+x^{2}}\right) \operatorname{erfc}(x) \mathrm{d} x
$$

Note that $i \operatorname{erf}(i x)$ is real. The following notation has also been used:

$$
\begin{aligned}
D & =4 \pi^{1 / 2} \frac{\hat{\omega}_{p}^{2}}{\hat{\omega}^{2}} \frac{a^{2}}{\hat{k}_{\perp}^{2}} \\
x_{n}^{2} & =\frac{a}{2}\left(\frac{n^{2}}{\hat{\omega}^{2}}-1\right) \\
\kappa & =\sqrt{2 / a} \hat{k}_{\perp}
\end{aligned}
$$

Note that in $A_{n}^{\mathrm{III}}, x_{n}^{2}<0$ since $\hat{\omega}>n$. Hence for a given $\hat{\omega}$ such that $m-1<\hat{\omega} \leq m$ for some integer $m \geq 1$, the dispersion relation is

$$
\begin{gathered}
\hat{\omega}^{2}-2 \hat{\omega}_{p}^{2}=D\left\{m^{4} A_{m}^{\mathrm{I}}+m^{4} A_{m}^{\mathrm{II}}+(m+1)^{4} A_{m+1}^{\mathrm{I}}+(m+1)^{4} A_{m+1}^{\mathrm{II}}+\ldots\right. \\
\left.+(m-1)^{4} A_{m-1}^{\mathrm{III}}+(m-2)^{4} A_{m-2}^{\mathrm{III}}+\ldots+A_{1}^{\mathrm{III}}\right\}
\end{gathered}
$$




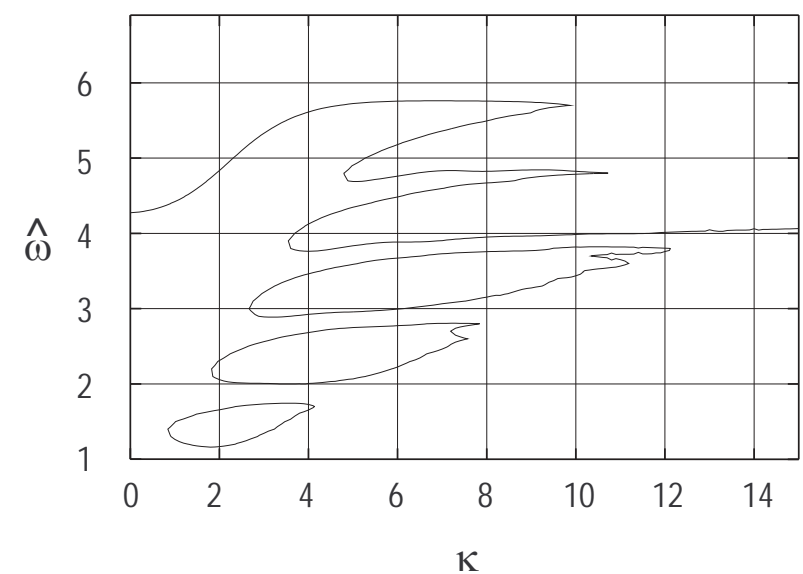

FIG. 3: Dispersion curves showing solutions in $\hat{\omega}-\kappa$ space for the case $a=10, \hat{\omega}_{p}=3$. Vertical axis is $\hat{\omega}$; horizontal axis is $\kappa$.

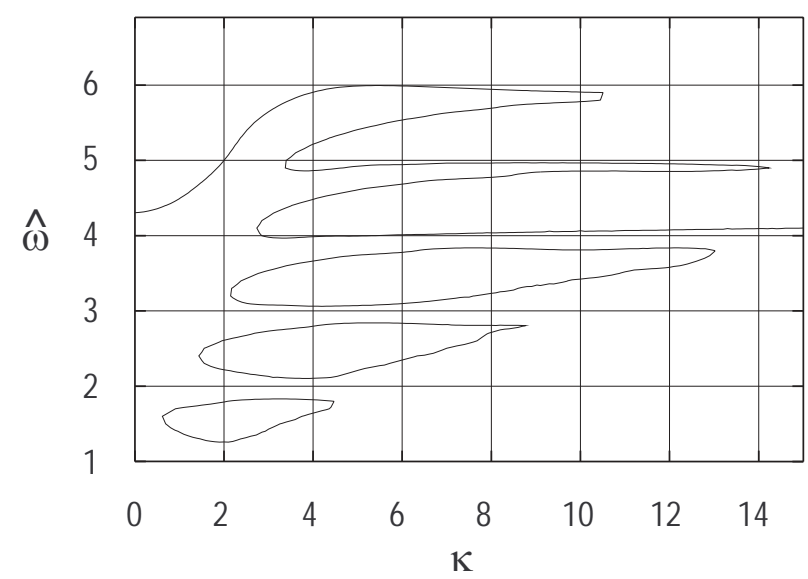

FIG. 4: Dispersion curves showing solutions in $\hat{\omega}-\kappa$ space for the case $a=20, \hat{\omega}_{p}=3$. Vertical axis is $\hat{\omega}$; horizontal axis is $\kappa$.

\section{A. General remarks}

The solutions to the dispersion relation are given in Figs. 3, 4 and 5, for the cases $a=10,20$ and 50 respectively. In each calculation, Bessel function terms up to 6 th order were included. The computer algebra system Macsyma[14] was used for all analytical and numerical manipulations. It is clear from these curves for the various values of $a$ that the weakly relativistic case is significantly different from the classical case. Not only are the classical single lines replaced by closed curves, there is only one solution for vanishingly-small $\hat{k}_{\perp}$. Associated with each set of dispersion curves is a minimum, non-zero value of $\kappa$, the latter increasing with increasing frequency. Moreover, there is also a maximum value of $\kappa$ for those solutions which form closed curves; once

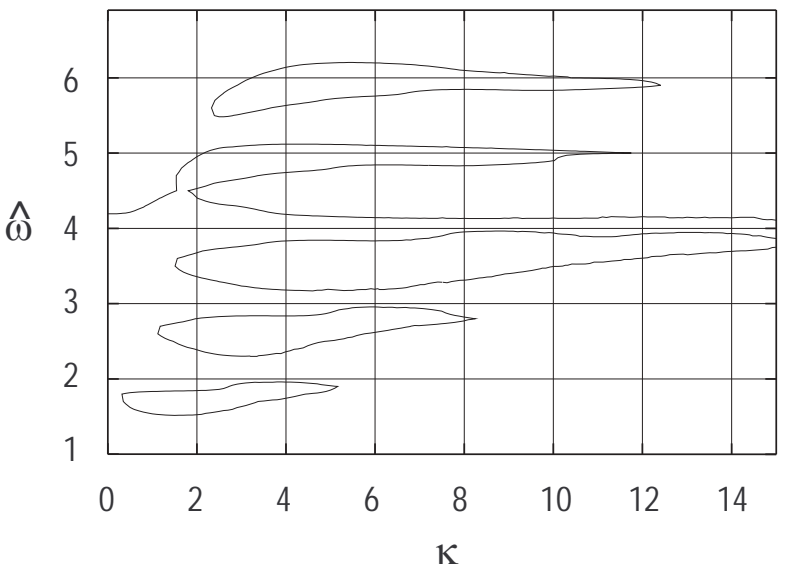

FIG. 5: Dispersion curves showing solutions in $\hat{\omega}-\kappa$ space for the case $a=50, \hat{\omega}_{p}=3$. Vertical axis is $\hat{\omega}$; horizontal axis is $\kappa$.

more, the higher the frequency, the greater the maximum valid $\kappa$. Notice also that solutions occur between the cyclotron harmonics. Earlier weakly relativistic modelling of electron-ion Bernstein modes revealed certain of these features $[6,8]$, including frequency down-shifting, finite minimum $k_{\perp}$ values and even the hint of island-type formations [8], albeit with complex wavenumbers.

However, not only are these treatments for electronion plasmas, and so unable to exploit the symmetry of the $e^{+} e^{-}$plasma, but they are also dependent on the binomial expansion of the relativistic $\gamma$, or on expansion in powers of $k_{\|}$, and so are of restricted validity.

In our article, the plasma is mass-symmetric, and the singularities appear in the full momentum integration. As a result, there are no complex roots to the dispersion relation for the specific case of perpendicular propagation. The treatment of residues given in the Appendix makes this clear.

The general appearance of the dispersion curves in this article reflects the fact that the surface which intersects the zero plane (that is, the plane in $\hat{\omega}-\hat{k}$ space where Eq. (41) is satisfied) to give the dispersion relations is not a singular one, as in the classical case; rather than the infinitely-thin slices produced by a singular surface piercing the zero-plane as it passes from $-\infty$ to $+\infty$, the dispersion curves are the zero contours of a well-behaved undulating surface. The singularities have been integrated out of the final form of the dispersion relation, resulting in the island shapes shown in Figs. 3, 4 and 5; note that the contouring algorithm is not flawless, and so there are minor imperfections present in the graphics.

Note also that as $a$ increases, the 'islands' elongate and drift upwards in frequency. The elongation admits smaller $\kappa$ solutions as each island stretches towards the frequency-axis intercept with $\kappa=0$, indicative of a closer agreement with the traditional classical case.

The next two sections address quantitatively the 
salient features of the dispersion curves.

\section{B. Properties of the integrals}

Note that $A_{n}^{\mathrm{I}}$ is negative definite, whilst $A_{n}^{\mathrm{II}}$ and $A_{n}^{\mathrm{III}}$ are positive definite. Given the strong dependence on index in the terms in the infinite sum, as is clearest in Eq. (53), any valid solution to the dispersion relation depends ultimately on balancing the positive and negative contributions from the $A_{n}^{\mathrm{I}, \mathrm{II}, \mathrm{III}}$, so that the resulting $\hat{\omega}^{2}>0$. The strongest dependence on $x_{n}$ is exhibited in $A_{n}^{\mathrm{I}}$ and $A_{n}^{\mathrm{III}}$, since in these integrals $x_{n}$ appears in the limits, and not just in the Bessel function arguments.

Consider the mean value theorem applied to $A_{n}^{\mathrm{I}}$ :

$$
A_{n}^{\mathrm{I}}=J_{n}^{2}\left(\kappa \sqrt{x_{n}^{2}-\xi^{2}}\right) \mathcal{E}_{\mathrm{I}}\left(x_{n}\right)
$$

where $\xi \in\left[0, x_{n}\right]$, and where the envelope $\mathcal{E}_{\mathrm{I}}\left(x_{n}\right)$ is defined to be

$$
\begin{aligned}
\mathcal{E}_{\mathrm{I}}(x) & =e^{-x^{2}} \int_{0}^{x} i \operatorname{erf}(i u) \mathrm{d} u \\
& =x e^{-x^{2}} i \operatorname{erf}(i x)+\pi^{-1 / 2}\left(1-e^{-x^{2}}\right)
\end{aligned}
$$

(In fact, $\mathcal{E}_{\mathrm{I}}$ is proportional to the Dawson function[15], and the entire analysis could be recast in Dawson function terms, rather than error function of imaginary argument.) Applying the same technique to the remaining integrals yields

$$
\begin{aligned}
A_{n}^{\mathrm{II}} & =J_{n}^{2}\left(\kappa \sqrt{x_{n}^{2}+\eta^{2}}\right) \mathcal{E}_{\mathrm{II}}\left(x_{n}\right) \\
\mathcal{E}_{\mathrm{II}}(x) & =\pi^{-1 / 2}
\end{aligned}
$$

and

$$
\begin{aligned}
A_{n}^{\mathrm{III}} & =J_{n}^{2}\left(\kappa \sqrt{x_{n}^{2}+\zeta^{2}}\right) \mathcal{E}_{\mathrm{III}}\left(\left|x_{n}\right|\right) \\
\mathcal{E}_{\mathrm{III}}(x) & =\pi^{-1 / 2}-x e^{x^{2}} \operatorname{erfc}(x)
\end{aligned}
$$

for some $\eta \geq 0, \zeta \geq\left|x_{n}\right|$ such that $0 \leq J_{n}^{2}\left(\kappa \sqrt{x_{n}^{2}-\eta^{2}}\right) \leq$ $\mu$, where $\mu$ is the maximum of $J_{n}^{2}(x)$ for $x \in[0, \infty)$, and $0 \leq J_{n}^{2}\left(\kappa \sqrt{x_{n}^{2}-\eta^{2}}\right) \leq \mu^{\prime}$, where $\mu^{\prime}$ is the maximum of $J_{n}^{2}(x)$ for $x \in\left[\left|x_{n}\right|, \infty\right)$. The envelopes $\mathcal{E}_{\mathrm{I}, \mathrm{III}}$ are shown in Fig. 6 , where it is clear that $\mathcal{E}_{\mathrm{I}}(x)$ has an extremum at $x_{c} \approx 1.16$, but that $\mathcal{E}_{\mathrm{III}}(x)$ is monotonically decreasing.

\section{Position and shape of dispersion curves}

Since it is important to balance the relative weights of the integrals, consider the implications of maximising the negative-definite contribution. For this to happen, $x_{n}$ must be at its critical value at which $\mathcal{E}_{\mathrm{I}}$ has greatest magnitude. In order to simplify the analysis, let this

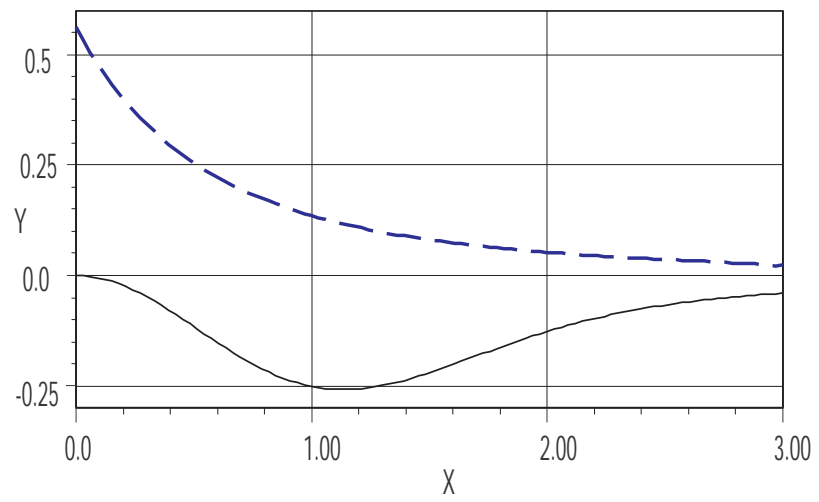

FIG. 6: Dashed curve shows $\mathcal{E}_{\text {III }}$ as a function of $x$; solid one is $\mathcal{E}_{\mathrm{I}}$.

correspond to $x_{n} \approx 1$, with the frequency $\hat{\omega}_{c}$ at which this occurs given by

$$
\hat{\omega}_{c}=n(1+2 / a)^{-1 / 2} \approx n(1-1 / a)
$$

Hence we expect solutions to the dispersion relation to be at frequencies below the classical cyclotron harmonic. Note that as $a \rightarrow \infty, \hat{\omega} \rightarrow n$, consistent with the classical case.

Since $\operatorname{erfc}(x) \approx 0$ for $x \gtrsim 4$, the range of the summations in Eq. (53) is limited, in practical terms. Thus in the simplest possible 'nearest neighbour' approximation, forming a balance of the negative and positive contributions means that $A_{n}^{\mathrm{I}}$ has to be comparable in magnitude to $A_{n-1}^{\mathrm{III}}$. If $x_{n} \approx 1$, then $x_{n-1} \approx 1-a / n<0$, assuming $n<a$. Consider only the envelope behaviour near the critical frequency, that is, consider $\hat{\omega}=\hat{\omega}_{c}(1+\delta)$, where $|\delta| \ll 1$. Regardless of the sign of $\delta$, if $\delta \neq 0, \mathcal{E}_{\mathrm{I}}$ falls below its maximum magnitude (being at an extremum). However, if $\delta>0$, then $\mathcal{E}_{\text {III }}$ also drops, but if $\delta<0$, $\mathcal{E}_{\text {III }}$ rises. Hence there is a finite range of $\omega$ over which the integrals $A_{n}^{\mathrm{I}}$ and $A_{n-1}^{\mathrm{III}}$ can maintain overall parity in contributions.

The envelope analysis must be complemented by assessing the Bessel function contribution, which of course contains the $\kappa$ variation. For $A_{n}^{\mathrm{I}}$, the argument to the Bessel function is

$$
\kappa\left(x_{n}^{2}-\xi^{2}\right)^{1 / 2}, \quad 0 \leq \xi \leq x_{n}
$$

and for $A_{n}^{\mathrm{III}}$, the Bessel argument is

$$
\kappa\left(x_{n}^{2}+\zeta^{2}\right)^{1 / 2}, \quad\left|x_{n}\right| \leq \zeta
$$

The position of the first zero $z_{n}$ of $J_{n}(z), n=1,2, \ldots$, increases with increasing order of the Bessel function, scaling approximately according to $z_{n} \approx 2.83+n$, with $z_{1} \approx 3.83$. Thus the full phase of the first peak of the Bessel function is sampled over a range of $\kappa \approx z_{n} / x_{n}$ for each $n$. This explains qualitatively why the dispersion 
curves form closed structures of increasing length as the harmonic number increases.

The Bessel function behaviour also controls the relative magnitudes of $A_{n}^{\mathrm{I}}$ and $A_{n}^{\mathrm{III}}$. Using small-argument expansions for the Bessel functions,

$$
\left|\frac{A_{n}^{\mathrm{I}}}{A_{n-1}^{\mathrm{III}}}\right| \sim \frac{1}{4} \frac{\kappa^{2}}{n^{2}} \frac{\left(x_{n}^{2}-\xi^{2}\right)^{n}}{\left(x_{n-1}^{2}+\zeta^{2}\right)^{n-1}}\left|\frac{\mathcal{E}_{\mathrm{I}}\left(x_{n}\right)}{\mathcal{E}_{\mathrm{III}}\left(x_{n-1}\right)}\right|
$$

Given that the optimum frequency is $\hat{\omega}_{c}$ for which the envelope of $A_{n}^{\mathrm{I}}$ is as large as it can be, Eq. (62) further constrains the dispersion curves to begin at larger values of $\kappa$ as $n$ increases, in order that the factor $\kappa / n$ does not erode the relative scaling necessary to maintain real solutions to the dispersion relation. This agrees well with the behaviour shown in Figs. 3, 4 and 5 in which the gradient of the line touching the left-hand edges of the islands in each of the cases $a=10,20$ and 50 is approximately $1,1.4$ and 2.3, consistent with the predicted behaviour $\sim a^{1 / 2}$.

\section{Behaviour for small $\hat{k}_{\perp}$}

In the limit of small $\hat{k}_{\perp}$, which is also the small $\kappa$ limit, the Bessel function argument can be expanded in order to find solutions that are the analogues of those in Section III A. Since $D$ contains $\hat{k}_{\perp}^{2}$ in the denominator, the zeroth-order contribution must come from the case $n=1$, with higher $n$ contributing to vanishingly small terms as $\hat{k}_{\perp} \rightarrow 0$. The hybrid resonance must come from $A_{1}^{\mathrm{III}}$, and so we have

$$
\begin{aligned}
A_{1}^{\mathrm{III}} & \approx e^{-x_{1}^{2}} \int_{\left|x_{1}\right|}^{\infty} \frac{1}{4} \kappa^{2}\left(x^{2}+x_{1}^{2}\right) \operatorname{erfc}(x) \mathrm{d} x \\
& =\frac{\kappa^{2}}{12} e^{-x_{1}^{2}}\left[\frac{1+2 x_{1}^{2}}{\sqrt{\pi}} e^{-\left|x_{1}\right|^{2}}-2\left|x_{1}\right| x_{1}^{2} \operatorname{erfc}\left(\left|x_{1}\right|\right)\right]
\end{aligned}
$$

The dispersion relation can then be approximated as

$$
\begin{aligned}
\hat{\omega}^{2} \approx & 2 \hat{\omega}_{p}^{2}+\frac{2}{3} a \sqrt{\pi} \frac{\hat{\omega}_{p}^{2}}{\hat{\omega}^{2}} \times \\
& {\left[\pi^{-1 / 2}\left(1+2 x_{1}^{2}\right)-2\left|x_{1}\right| x_{1}^{2} e^{-x_{1}^{2}} \operatorname{erfc}\left(\left|x_{1}\right|\right)\right] }
\end{aligned}
$$

The asymptotic expansion[15]

$$
\operatorname{erfc}(z) \sim \frac{e^{-z^{2}}}{\sqrt{\pi} z}\left(1-\frac{1}{2 z^{2}}+\frac{3}{4 z^{4}} \ldots\right)
$$

when substituted into Eq. (64) yields

$$
\hat{\omega}^{2}-2 \hat{\omega}_{p}^{2} \approx \frac{2 \hat{\omega}_{p}^{2}}{\hat{\omega}^{2}-1}
$$

which on rearrangement yields the expected hybrid resonance as the non-trivial solution:

$$
\hat{\omega}^{2} \approx 1+2 \hat{\omega}_{p}^{2}
$$

Notice that when the same approximations are made in the context of $\hat{\omega} \leq 1$ we have

$$
A_{1}^{\mathrm{I}}+A_{1}^{\mathrm{II}} \approx \frac{\kappa^{2}}{12}\left[2 x_{1}^{3} e^{-x_{1}^{2}} i \operatorname{erf}\left(i x_{1}\right)+\pi^{-1 / 2}\left(2 x_{1}^{2}+1\right)\right]
$$

The required asymptotic expansion is now for $\operatorname{erf}(i x)$. This is readily obtained via the hypergeometric function[15]

$$
\operatorname{erf}(z)=\frac{2 z}{\sqrt{\pi}} M\left(\frac{1}{2}, \frac{3}{2},-z^{2}\right)
$$

using which yields

$$
\operatorname{erf}(i x) \sim i \frac{e^{x^{2}}}{\sqrt{\pi} x}\left(1+\frac{1}{2 x^{2}}+\frac{3}{4 x^{4}} \cdots\right)
$$

and so in the asymptotic limit,

$$
\hat{\omega}^{2}-2 \hat{\omega}_{p}^{2} \approx-\frac{\hat{\omega}_{p}^{2}}{\hat{\omega}^{2}} \frac{a}{x_{1}^{2}}
$$

which is the same as Eq.(66). The only possible solution is the trivial one, $\hat{\omega}=0$.

Note that the special case of $x_{1}=0$ is not a solution, since $\hat{\omega}=1$ is not a solution. In general, $x_{n}=0$ means that $A_{n}^{\mathrm{I}}=0$, and $A_{n}^{\mathrm{II}}$ is identical to $A_{n}^{\mathrm{III}}$.

In summary, there is only one solution to the dispersion relation for the case $\hat{k}_{\perp} \rightarrow 0$, namely the mode which becomes the hybrid resonance at $\kappa=0$. This contrasts markedly with the classical treatment, in which all the cyclotron frequencies were solutions (save the one immediately below the hybrid, and the fundamental). Note also that for each value of $\kappa$, there are twice as many frequencies corresponding to solutions of the dispersion relation than there are in the classical case, reflecting the continuity of the general surface (of which the zero contour yields the dispersion curves), and therefore the undulations above and below the zero plane will yield two intersections, rather than one. Note also that the band-gaps which appear above the hybrid in the classical case persist everywhere in the weakly relativistic one. No single mode has an arbitrarily large range of $\kappa$ for a given small frequency range, as in the classical case.

\section{E. Trapped modes}

It is clear from the weakly relativistic dispersion curves that there are a number of modes with vanishing group velocity, that is, for which the tangent to the normalised $\omega-k_{\perp}$ curve is zero. Although the classical case also had many such modes above the hybrid frequency, The 
weakly relativistic case has two solutions between each cyclotron harmonic, for those dispersion solutions that form closed curves. Moreover, such stationary modes are present at frequencies below the hybrid. Hence a non-localised, broad-band disturbance of such a plasma would yield many propagating electrostatic waves, and several non-propagating stationary electrostatic oscillations. Given that plasma oscillations can be a source of electromagnetic radiation in pair-plasmas[16, 17] it is significant that the spectrum of possible electrostatic modes is more richly structured in the relativistic case than the classical one.

\section{DISCUSSION}

In this paper we have addressed the kinetic theory of weakly relativistic electron-positron plasmas, producing dispersion relations for the electrostatic Bernstein modes. The treatment presented here preserves the full momentum dependence of the cyclotron frequency, albeit with a relaxation on the true relativistic form of the distribution function. The form of the dispersion curves is markedly different from that of the well-known classical singular dispersion relations, in that the $\omega-k_{\perp}$ relations form closed, island domains, between the cyclotron harmonics, and show band-gaps distributed throughout the solution space, rather than confined to frequencies just above the hybrid. Moreover, there are extra limitations on allowed modes for long and short wavelengths, and within these limitations, there are greater numbers of allowed $\omega-k_{\perp}$ modes than in the classical case. Some of this new structure arises from the mass symmetry, but most derives from careful handling of the integrations in momentum space.

The implications of this new treatment are confined largely to astrophysical plasmas, where relativistic electron-positron plasmas occur naturally. It is very unlikely that the results presented here impact on any laboratory based electron-ion plasma, in which the relative mobility of the species is paramount.

For example, in a pulsar atmosphere, the spectrum of propagating electrostatic waves is more structured than implied by the classical case. Waves are significantly more band-width limited, and there is a greater number of trapped modes. These effects will have to be taken into account in any future treatment of the radiation from such sources.

The theoretical approach detailed in this article is also valid for the ordinary and extraordinary electromagnetic modes in equal-mass plasmas, including Landau damping.

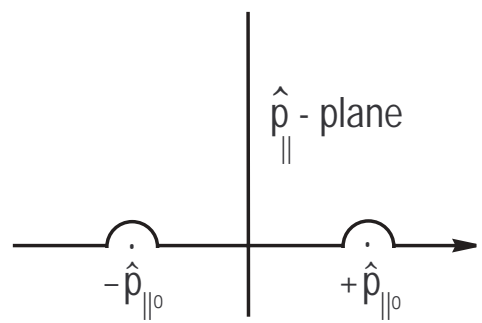

FIG. 7: The Bromwich contour for the $\hat{p}_{\|}$integration

\section{APPENDIX A: HANDLING OF SINGULAR INTEGRALS}

A proper derivation of the dispersion relation Eq. (46), and especially the correct handling of the singularities in the integrals Eq. (44) and Eq. (45), requires the time dependence to be treated as an initial value problem. As is well-known, this was first pointed out by Landau in his derivation of the damping of electrostatic waves in an unmagnetised plasma (so-called Landau damping). The presence of a uniform magnetic field gives rise, in the classical, non-relativistic case, to undamped electrostatic Bernstein waves in a plasma with a stationary ion background, and as is shown in section 2.1 the extension to a classical electron-positron plasma leads to similar conclusions.

In the case of weakly relativistic effects, of primary interest in this paper, singularities arise where the denominator of the $\hat{p}_{\|}$integral vanishes. In the derivation of Eq. (9) we introduced the perturbation $\operatorname{expi}(\boldsymbol{k} \cdot \boldsymbol{r}-\omega t)$. We note here that strictly the frequency $\omega$ should be introduced by a Laplace transform, in which $\omega$ has a positive imaginary part, and the first-order distribution function $f$ and electric field $\boldsymbol{E}$ have given initial values at $t=0$. The subsequent behaviour of the plasma is then to be obtained by forming the inverse Laplace transform of the electric field. The presence of a positive imaginary part in $\omega$ implies that the $\hat{p}_{\|}$integration is only defined in the upper half $\omega$-plane, and therefore no singularities actually occur. In order to investigate the properties of the dispersion relation Eq. (46) for all values of $\omega$, and in particular for real $\omega$, Eq. (46) must be analytically continued into the region where $\operatorname{Im}(\omega) \leq 0$. This is achieved by treating $\hat{p}_{\|}$as a complex variable and displacing the path of integration so that it lies on the real axis of $\hat{p}_{\|}$, except at the singular points $\pm \hat{p}_{\| 0}$ where the path of integration is indented above the real axis, as in Fig. 7. 


\section{Case (i): $\mathcal{I}_{1}(\alpha, \beta)$}

The treatment of the integral

$$
\mathcal{I}_{1}(\alpha, \beta)=\int_{-\infty}^{\infty} \mathrm{d} z \frac{\exp \left(-\alpha z^{2}\right)}{z^{2}-\beta^{2}}
$$

requires the definition of a suitable integration contour in order that the integral is meaningful at the poles $z= \pm \beta$. Taking the contour as shown in Fig. 7, it is immediately apparent that the residues around each pole are equal and opposite in sign, leaving the contour integral equal to the principal part along the real axis:

$$
\begin{gathered}
\mathcal{I}_{1}(\alpha, \beta)=\frac{1}{\beta} \mathcal{P} \int_{-\infty}^{\infty} \frac{\exp \left(-\alpha \beta^{2} u^{2}\right)}{u^{2}-1} \mathrm{~d} u \\
=\mathcal{A}\left(\alpha \beta^{2}\right) / \beta
\end{gathered}
$$

where $z=\beta u$. Notice that

$$
\frac{\partial \mathcal{A}(x)}{\partial x}+\mathcal{A}(x)=-\int_{-\infty}^{\infty} \exp \left(-x u^{2}\right) \mathrm{d} u=-\sqrt{\frac{\pi}{x}}
$$

Given that

$$
\mathcal{P} \int_{-\infty}^{\infty} \frac{\mathrm{d} u}{u^{2}-1}=0
$$

the full solution to Eq. (A4)is

$$
\mathcal{A}(x)=\mathrm{i} \pi e^{-x} \operatorname{erf}(\mathrm{i} \sqrt{x})
$$

2. Case (ii): $\mathcal{I}_{2}(\alpha, \beta)$

This time the integral has no poles along the real axis:

$$
\begin{aligned}
& \mathcal{I}_{2}(\alpha, \delta) \quad=\int_{-\infty}^{\infty} \frac{\exp \left(-\alpha z^{2}\right)}{z^{2}+\delta^{2}} \mathrm{~d} z \\
&=\frac{1}{\delta} \int_{-\infty}^{\infty} \frac{\exp \left(-\alpha \delta^{2} u^{2}\right)}{u^{2}+1} \mathrm{~d} u \\
&=\mathcal{B}\left(\alpha \delta^{2}\right) / \delta
\end{aligned}
$$

Proceeding as before, $\mathcal{B}$ satisfies the differential equation

$$
\frac{\partial \mathcal{B}(x)}{\partial x}-\mathcal{B}+\sqrt{\frac{\pi}{x}}=0
$$

which, together with

$$
\int_{-\infty}^{\infty} \frac{\mathrm{d} u}{1+u^{2}}=\pi
$$

yields the solution for $\mathcal{B}(x)$ as

$$
\mathcal{B}(x)=\pi e^{x} \operatorname{erfc}(\sqrt{x})
$$

[1] A. P. Lightman, Astrophys. J.253, 842 (1982)

[2] A. Georgiou, Plasma Phys. Control. Fusion 38, 347 (1996)

[3] I. B. Bernstein, Phys. Rev. 109, 10 (1958)

[4] F. W. Crawford, Nucl. Fusion 5, 73 (1965)

[5] T. H. Stix, Waves in Plasmas,(AIP Press, New York, 1992)

[6] P. A. Robinson, J. Plasma Phys. 37, 435 (1987); 37, 449 (1987)

[7] Yu. n. Dnestrovskii and D. P. Kostomarov, Sov. Phys. JETP 13, 986 (1961)

[8] I. P. Shkarofsky, Phys. Fluids 9, 570 (1966)

[9] B. Buti, Phys. Fluids. 6, 89 (1963)

[10] A. A. Mamun, Phys. Plasmas 1, 2096 (1994)

[11] D. C. Montgomery and D. A. Tidman, Plasma Kinetic
Theory, (McGraw-Hill, New York, 1964)

[12] N. A. Krall and A. W. Trivelpiece, Principles of plasma physics, (McGraw-Hill, New York, 1973)

[13] D. J. Rose and M. Clark, Plasmas and Controlled Fusion, (MIT Press, 1961)

[14] Macsyma Symbolic/numeric/graphical mathematics software, version 2.1 for PC.

[15] M. Abramowitz and I. Stegun, Handbook of Mathematical Functions (Dover, New York, 1972)

[16] D. A. Diver, A. A. da Costa, and E. W. Laing, Astron. Astrophys. 387, 339 (2002)

[17] T. Kitanishi, J.-I. Sakai, K.-I. Nishikawa and J. Zhao, Phys. Rev. E 53, 6376 (1996) 